distance (Table 1). Only numerical improvement in IG compared to numerical deterioration in CG, which have not reached statistical significance, were observed in patient reported outcomes (CHFS, MHISS, HAQ, SHAQ, SF-36).

Conclusions: Our physiotherapy program not only prevented the natural course of progressive deterioration of function of hands/mouth (observed in the control group), but led to a significant improvement in monitored parameters, which was clinically meaningful in a substantial proportion of patients.

Acknowledgements: Supported by AZV-16-33574A.

Disclosure of Interest: None declared

DOI: 10.1136/annrheumdis-2017-eular.4875

\section{FRI0400 EFFICACY OF INTRAVENOUS IMMUNOGLOBULIN THERAPY IN REFRACTORY DYSPHAGIA IN PATIENTS WITH IDIOPATHIC INFLAMMATORY MYOPATHIES}

M. Giannini ${ }^{1}$, M.L. Fiorella ${ }^{2}$, D. D'Abbicco ${ }^{3}$, A. Amati ${ }^{4}$, A. Lia ${ }^{4}$, F. Girolamo ${ }^{4}$,

F. Cacciapaglia ${ }^{1}$, F. lannone ${ }^{1} .{ }^{1}$ Dipartimento di Emergenza e Trapianti di Organi - Sezione di Reumatologia; ${ }^{2}$ Dipartimento di Scienze mediche di base, neuroscienze ed organi di senso - Clinica Otorinolaringoiatrica; ${ }^{3}$ Dipartimento di Emergenza e Trapianti di Organi - Chirurgia Marinaccio; ${ }^{4}$ Dipartimento di Scienze mediche di base, neuroscienze ed organi di senso - Sezione di Neurologia, Università degli Studi di Bari, Bari, Italy

Background: Polymyositis (PM) and Dermatomyositis (DM) are systemic inflammatory diseases affecting skeletal muscles and other organs, particularly hypopharynx and the upper tract of the esophagus, causing motility dysfunction. The current treatment for PM and DM relies on steroids and immunosuppressants, but esophageal involvement is often refractory to standard therapies.

Objectives: To assess the efficacy and safety of intravenous immunoglobulins (Ig-iv) as therapy of refractory dysphagia (1) in patients affected with PM/DM who have received conventional treatments for at least three months. These are the extended data of a previous abstract entitled "Improvement of refractory dysphagia in patients with idiopathic inflammatory myopathies receiving immunoglobulin intravenous therapy" accepted to EULAR 2015 Congress.

Methods: We enrolled 17 patients (15 F), 8 with DM, 9 with PM according to Bohan and Peter criteria, treated with prednisone (mean dose $20 \mathrm{mg} /$ day) \pm Methotrexate $(15 \mathrm{mg} / \mathrm{wk})$ or Azathioprine (100 mg/day) for at least 3 months, without improvement of dysphagia. All patients received one infusion of $\lg$ at a standard dose $(2 \mathrm{~g} / \mathrm{kg}$ in a day) every two months for 3 cycles. In all patients, at entry and before each infusion, creatine kinase (CK) levels and Manual Muscle strength Test on 12 muscle groups (MMT12) were evaluated. Dysphagia assessment included: Eating Assessment Tool (EAT) impaired for score $\geq 3$; Three-oz Swallow Test (30ST) and fibrolaryngoscopy (FEES) for pharyngeal muscle propulsion and stasis of liquid/solid bolus detection. Student T-test and Chi-square test were used to compare baseline and follow-up data.

Results: At entry, all patients had an EAT score $\geq 3$ with a mean score of $16.2 \pm 9$, 3 patients presented a 3oST for solid (STPNS) impaired, and only 1 patient also for liquid (STPNL). CK levels were slightly increased (474 $\pm 720 \mathrm{UI} / \mathrm{L})$, and MMT12 score was $4.45 \pm 0.35$. Following Ig-iv therapy we observed a significant improvement of EAT score after the first dose $(9 \pm 8, p=0.006)$ with a further reduction after the second dose $(7.4 \pm 8, p=0.007)$ and stability after the third one $(7.6 \pm 8, p=0.008)$. An improvement of pharyngeal muscle propulsion, with a significant resolution of solid stasis and a partial resolution of liquid stasis were observed. In addition, MMT12 score progressively improved after each dose, increasing at $4.86 \pm 0.16$ after the third dose $(p=0.001)$. Noteworthy, after the third Ig-iv dose, three patients stopped the steroid and the remaining 12 were treated with a significant lower dose of $5 \mathrm{mg} /$ day of prednisone $(\mathrm{p}=0.04)$.

Conclusions: According to literature, our findings provided evidence that Ig-iv induced a meaningful improvement of dysphagia, also having a steroid-sparing effect. An improvement of muscle strength was also detected. Although further studies of larger cohort of patients and a longer follow-up time are needed, our findings corroborate the utility of Ig-iv in the treatment of more resistant idiopathic inflammatory myositis related dysphagia.

\section{References:}

[1] Marie I et al. Intravenous immunoglobulins for steroid-refractory esophageal involvement related to polymyositis and dermatomyositis: a series of 73 patients. Arthritis Care Res. 2010;62(12).

Disclosure of Interest: None declared

DOI: 10.1136/annrheumdis-2017-eular.5049

\section{FRI0401 INITIAL MANIFESTATION DETERMINES CLINICAL ENTITY AND COURSE IN PATIENTS WITH ANTI-CENTROMERE ANTIBODY: A SINGLE CENTER LONGITUDINAL RETROSPECTIVE COHORT STUDY}

M. Tsukamoto ${ }^{1,2}$, K. Suzuki ${ }^{1}$, N. Seta ${ }^{2}$, T. Takeuchi ${ }^{1}$. ${ }^{1}$ Division of Rheumatology, Department of Internal Medicine, Keio University School of Medicine, Tokyo; ${ }^{2}$ Department of Internal Medicine, Tokyo Dental College Ichikawa General Hospital, Ichikawa, Japan

Background: Anti-centromere antibody (ACA) is often detected in sera from autoimmune diseases, such as limited cutaneous systemic sclerosis (SSc) and primary biliary cirrhosis (PBC). ACA-positive Sjögren's syndrome (SjS) is recently considered as a distinct clinical subgroup in SiS [1-2]. The other autoimmune diseases associated with ACA-positive have not fully elucidated and the role of ACA in their pathogenesis still remains unclear. In addition, comprehensive and enough clinical information from the point of view of ACA positive cases has not been accumulated.

Objectives: To clarify clinical features of patients with ACA and their association with autoantibodies.

Methods: Patients with discrete-speckled pattern in anti-nuclear antibody (ANA) test and/or positive ACA, who visited to our department during over 20 years between May 1995 and December 2016, were enrolled. Clinical information and immunological tests including immunoglobulin (Ig) and serum autoantibodies were collected and statistically analyzed.

Results: Discrete-speckled pattern in ANA test and/or positive ACA were identified in 309 patients. There were 292 female and 17 male and the average age was 60 years old. Proportion of concomitant ANA patterns were speckled (16\%), homogenous (7\%), cytoplasmic (3\%) and/or nucleolar (3\%) pattern. 186 patients $(60 \%)$ had Raynaud's phenomenon and 149 patients $(48 \%)$ had sclerodactyly. 162 patients $(52 \%)$ had oral and/or ocular dryness symptoms. 21 patients $(6 \%)$ had interstitial pneumonia and 13 patients $(4 \%)$ had pulmonary hypertension. 42 patients $(13 \%)$ had arthritis. 19 patients $(6 \%)$ had thyroid disease. 214 patients $(69 \%)$ were classified into 17 autoimmune diseases (including overlap cases) from symptoms at initial visit, while other 95 patients (31\%) who did not meet the criteria were not diagnosed. $\lg$, $\lg M$ and $\lg A$ in the patients who were classified into autoimmune diseases were higher than that in other patients who were not classified ( $p=0.0007, p=0.0057$ and $p=0.0158$, respectively). In the 214 patients $(69 \%)$, there were most of patients who were diagnosed as SSc $(n=113,36 \%)$ and/or SjS ( $n=78,25 \%) .25$ patients $(8 \%)$ and 22 patients $(7 \%)$ were diagnosed with rheumatoid arthritis (RA) and PBC, respectively. On the other hand, the patients who diagnosed as systemic lupus erythematosus were $3(1 \%)$, and nobody was diagnosed as myositis. The mean observation period was 80 months. 2 patients in 95 patients who were unclassified at initial visit were diagnosed as RA or autoimmune hepatitis by developing new symptoms during observation. A patient was newly diagnosed as SSc in addition to SjS because sclerodactyly was newly developed. In other patients, the diagnosis was not changed and added during observation.

Conclusions: Our single center longitudinal retrospective cohort study confirmed that ACA-positive cases were classified into various types of autoimmune disease. Few patients had new symptoms during observation but the initial diagnosis was unchanged. Our data demonstrated that initial manifestation determined clinical entity and course in patients with ACA.

References:

[1] J Rheumatol 2001; 28: 2238.

[2] Int J Rheum Dis 2015; $18: 776$.

Disclosure of Interest: None declared

DOI: 10.1136/annrheumdis-2017-eular.5692

\section{FRI0402 ONSET CLINICAL INVOLVEMENT AS PROGNOSTIC FACTOR IN SYSTEMIC SCLEROSIS (SSC)}

M. Pestaña Fernández ${ }^{1}$, M. Rubio Rivas ${ }^{1}$, C. Tolosa Vilella ${ }^{2}$, A. Guillén del Castillo $^{3}$, D. Colunga Argüelles ${ }^{4}$, L. Trapiella Martinez ${ }^{5}$, V. Fonollosa Pla ${ }^{3}$, C.P. Simeón Aznar ${ }^{3}$ on behalf of RESCLE. ${ }^{1}$ Internal Medicine Department, Hospital Universitario de Bellvitge, Barcelona: ${ }^{2}$ Internal Medicine Department, Corporación Sanitaria Universitaria Parc Taulí, Sabadell; ${ }^{3}$ Autoinmune Diseases Unit. Internal Medicine Department, Hospital Vall d'Hebron, Barcelona; ${ }^{4}$ Internal Medicine Department, Hospital Universitario Central de Asturias; ${ }^{5}$ Internal Medicine Department, Hospital de Cabueñes, Oviedo, Spain

Background: In most patients with SSc, the onset is in the form of Raynaud's phenomenon (RP), but in a few is in the form of visceral or musculoskeletal involvement or skin sclerosis. Several risk factors are related to poor outcome in patients with SSc, but never before has the mode of onset been described as one of them.

Objectives: To asses the mode of onset as prognostic factor in patients with SSc. Methods: A retrospective and multicentre cohort study including 1625 patients with SSc, based on the National Spanish Scleroderma Registry (RESCLE) which includes 28 referral centres.

RP, puffy hands, arthralgia/arthritis, skin sclerosis, intersititial lung disease (ILD), pulmonary arterial hypertension (PAH) and esophageal hipomotility where included as clinical modes of onset

Results: The cohort was split up in two groups: 1342 patients (83\%) presented as RP and $283(17 \%)$ presented as non-RP (see graphic below)

Non-RP patients where younger at onset of disease ( $44.7 \pm 16.5$ vs $49 \pm 15.6$ years, $p<0.001$ ), showed higher percentage of diffuse subtipe ( $36 \%$ vs $19 \%, p<0.001$ ), developed more visceral involvement during the course of the illness and where more frequently anti-RNAp III positive (29 vs $10 \%, p<0.001)$.

Overall mortality was $16 \%$ in patients presented as RP vs $23 \%$ in non-RP patients $(p=0.003)$. Few differences where noted among causes of death related to SSc, but the higher mortality was observed in RP group due to PAH (19\% vs $8.1 \%$, $\mathrm{p}=0.05)$ and due to ILD in non-RP group ( $24 \%$ vs $8.3 \%, p=0.002)$.

Survival of patients with RP at onset was better at anytime than those with a non-RP onset: 0.97 vs. 0.89 at 5 years, 0.93 vs. 0.82 at 10 years, 0.83 vs. 0.63 at 20 years and 0.71 vs. 0.51 at 30 years from onset $(p<0.001)$. 